\title{
The uncertainty measure for $q$-exponential distribution function
}

\author{
OU CongJie ${ }^{1,2^{*}}$, EL KAABOUCHI Aziz ${ }^{2}$, WANG QiuPing Alexandre ${ }^{2}$ \& CHEN JinCan ${ }^{2,3}$ \\ ${ }^{1}$ College of Information Science and Engineering, Huaqiao University, Xiamen 361021, China; \\ ${ }^{2}$ Institut Supérieur des Matériaux et Mécaniques Avancés du Mans, 44, Avenue F.A. Bartholdi, 72000 Le Mans, France; \\ ${ }^{3}$ Department of Physics, Xiamen University, Xiamen 361005, China
}

Received April 13, 2012; accepted June 10, 2012

\begin{abstract}
Based on the $q$-exponential distribution which has been observed in more and more physical systems, the uncertainty measure of such an abnormal distribution can be derived by employing a variational relationship which can be traced from the first and second thermodynamic laws. The uncertainty measure obtained here can be considered as the entropic form for the abnormal physical systems having observable $q$-exponential distribution. This entropy will tend to the Boltzmann-Gibbs entropy when the nonextensive parameter tends to unity. It is very important to find that this entropic form is always concave and the systemic entropy is maximizable.
\end{abstract}

q-exponential distribution, VarEntropy method, MaxEnt, nonextensive entropy

Citation: $\quad$ Ou C J, El Kaabouchi A, Wang Q A, et al. The uncertainty measure for $q$-exponential distribution function. Chin Sci Bull, 2013, 58: 1524-1528, doi: $10.1007 / \mathrm{s} 11434-012-5664-3$

In classical statistical mechanics, the Boltzmann factor is very important to discuss the thermo dynamical properties of the physical systems. This factor is obtained under some ideal conditions, e.g. there are no other interactions except the elastic collisions between the particles of the system and the number of particles tends to infinity. However, with the development of modern physics, these ideal conditions become more and more imprecise and the classical theory should be generalized. In fact, if the internal interactions are taken into account, the probability distributions, $p(x) \propto[1-(1-\tilde{q}) x]^{1 /(1-\tilde{q})} \equiv e_{\tilde{q}}(-x)$, can be observed in more and more physical systems [1-6], where $x$ represents the observable quantity, such as particles' displacement [4,5], atomic momentum [6], systemic Hamiltonian [7,8], and $\tilde{q}$ can be considered as a nonextensive parameter. This distribution function is called the $q$-exponential distribution function and the corresponding generalization of classical theory is named nonextensive statistical mechanics. Obviously, the $q$-exponential factor $e_{\tilde{q}}(-x)$ will converge to the Boltz-

*Corresponding author (email: jcou@ @qu.edu.cn) mann factor if $\tilde{q}$ tends to 1 .

On the other hand, according to the maximum entropy principle (MaxEnt), the probability distribution function of the system can be determined by the Lagrange multiplier method. In this method, the entropic form as well as the constraints of probability normalization condition and the expectations of the observable quantities dominate the probability distribution. Obviously, the well known Boltzmann-Gibbs entropy $\left(S_{\mathrm{BG}}=-k \sum_{i=1}^{W} p_{i} \ln p_{i}\right)$ associated with the constraints of the probability normalization $\sum_{i} p_{i}=1$ and normal expectation $\langle O\rangle=\sum_{i} p_{i} O_{i}$ cannot yield the $q$-exponential distribution mentioned above, where $O_{i}$ 's represent the $W$ possible values of the observable quantity. By the same trick, several different nonextensive entropies can be substituted and the corresponding results are listed as follows. For the sake of convenience, we set the Boltzmann constant $k=1$ below.

$$
S_{\tilde{q}}^{\mathrm{T}}=\frac{1-\sum_{i=1}^{W} p_{i}^{\tilde{q}}}{\tilde{q}-1},
$$




$$
S_{\tilde{q}}^{\mathrm{NT}}=\frac{1}{\tilde{q}-1}\left(\frac{1}{\sum_{i=1}^{W} p_{i}^{\tilde{q}}}-1\right)
$$

and

$$
S_{\tilde{q}}^{\mathrm{R}}=\frac{\ln \left(\sum_{i=1}^{W} p_{i}^{\tilde{q}}\right)}{1-\tilde{q}}
$$

are the Tsallis entropy $[9,10]$ normalized Tsallis entropy $[11,12]$ and Renyi entropy [13], respectively. All of these three entropies will converge to the Boltzmann-Gibbs one at the $\tilde{q} \rightarrow 1$ limit. The functional of the Lagrange multiplier method can be written as

$$
\begin{aligned}
L^{J}\left(\left\{p_{i}\right\}, \alpha, \beta\right)= & S^{J}\left(\left\{p_{i}\right\}\right)-\alpha \sum_{i} p_{i} \\
& -\beta \sum_{i} p_{i} O_{i} \quad(J=\mathrm{T}, \mathrm{NT}, \mathrm{R}),
\end{aligned}
$$

where $\alpha$ and $\beta$ are the Lagrange multipliers associated with the probability normalization condition and expectation value. Variation of eq. (4) with respect to $p_{i}$ yields the following probability distributions:

$$
\begin{gathered}
p_{i}^{\mathrm{T}}=\left(\sum\left(p_{j}^{T}\right)^{\tilde{q}}\right)^{\frac{1}{\tilde{q}-1}}\left\{1-\frac{\tilde{q}-1}{\tilde{q}} \beta \frac{O_{i}-\langle O\rangle}{\sum\left(p_{j}^{T}\right)^{\tilde{q}}}\right\}_{+}^{\frac{1}{\tilde{q}-1}}, \\
p_{i}^{\mathrm{NT}}=\left(\sum\left(p_{j}^{\mathrm{NT}}\right)^{\tilde{q}}\right)^{\frac{1}{\tilde{q}-1}}\left\{1-\frac{\tilde{q}-1}{\tilde{q}} \beta\left(O_{i}-\langle O\rangle\right) \sum\left(p_{j}^{T}\right)^{\tilde{q}}\right\}_{+}^{\frac{1}{\tilde{q}-1}},
\end{gathered}
$$

and

$$
p_{i}{ }^{\mathrm{R}}=\left(\sum\left(p_{j}^{R}\right)^{\tilde{q}}\right)^{\frac{1}{\tilde{q}-1}}\left\{1-\frac{\tilde{q}-1}{\tilde{q}} \beta\left(O_{i}-\langle O\rangle\right)\right\}_{+}^{\frac{1}{\tilde{q}-1}}
$$

where $\{\mu\}_{+} \equiv \max \{0, \mu\}$. From eqs. (5)-(7) it can be seen that none of them can be transformed to the observed $q$-exponential distribution since $(\tilde{q}-1) / \tilde{q}$ is very different from $(1-\tilde{q})$ or $(\tilde{q}-1)$ unless $\tilde{q} \rightarrow 1$. In many literatures the escort expectation $\left(\langle O\rangle=\sum_{i} p_{i}^{\tilde{q}} O_{i} / \sum_{j} p_{j}^{\tilde{q}}\right)$ has been adopted to derive the probability distribution. However, it has been proven that the escort expectation is neither stable [14] nor in consistent with the generalized Stosszahlansatz and the associated $H$-theorem [15]. It means that this kind of expectation is not experimental robustness or observable so the corresponding results cannot be compared with the experimental observations. It is worth to mention that in [10] the authors have obtained exactly the $q$-expectation function by maximizing the Tsallis entropy with the unnormalized expectation $\left(\langle O\rangle=\sum_{i} p_{i}^{\tilde{q}} O_{i}\right)$. However, this definition is in conflict with the expectation of a constant $C_{1}$, i.e. $C_{1} \neq \sum_{i} p_{i}^{\tilde{q}} C_{1} \quad(\tilde{q} \neq 1)$. Thus, this method is seldom adopted at present. Furthermore, maximizing the incomplete entropy [16] by the Lagrange multiplier method also yields a distribution function [17] that is different from the $q$-exponential function mentioned above. To sum up, all the nonextensive entropies mentioned above cannot yield the $q$-exponential distribution function under reasonable constraints using the standard Lagrange multiplier method.

Recently, a variational relationship (VarEntropy method) between the random variables and the uncertainty measure of the system has been proposed [18] to discuss the probability distribution function of the system. It is worth to point out that the uncertainty measure can be considered as the entropy of the system [19,20] since the "VarEntropy" is exactly the reverse process of "MaxEnt" [21]. In principle, one can get different entropic forms for various distribution functions by using the VarEntropy method. In the present paper, the VarEntropy method is adopted to investigate the uncertainty measure of the $q$-exponential distribution and some properties of this entropic form are discussed.

\section{VarEntropy method for $q$-exponential dis- tribution function}

The variational relationship between a random variable $x$ of the system and the uncertainty measure can be written as [18]

$$
\mathrm{d} I=\mathrm{d}\langle x\rangle-\langle\mathrm{d} x\rangle=\sum_{i=1}^{W} x_{i} \mathrm{~d} p_{i}
$$

where " \langle\rangle " means the normal expectation, i.e. $\langle x\rangle=\sum_{i} p_{i} x_{i}$. The $q$-exponential distribution can be written as

$$
p_{i}=\frac{1}{Z}\left[1-(1-\tilde{q}) x_{i}\right]^{1 /(1-\tilde{q})} .
$$

Eq. (9) has another symmetrical form:

$$
p_{i}=\frac{1}{Z}\left[1-(q-1) x_{i}\right]^{1 /(q-1)},
$$

if one sets $q=2-\tilde{q}$. The partition function $Z$ can be written as

$$
Z=\sum_{i=1}^{W}\left[1-(q-1) x_{i}\right]^{1 /(q-1)} .
$$

The value of $Z$ depends on the random variable distribution $\left\{x_{i}\right\}$ and the nonexetensive parameter $q$ so that, in general, it will not equal to 1 . However, if a spectrum shift $\left(x_{i}\right.$ $\left.\rightarrow x_{i}-a\right)$ is adopted, it is possible to obtain

$$
Z=\sum_{i=1}^{W}\left[1-(q-1)\left(x_{i}-a\right)\right]^{1 /(q-1)}=1 \quad(q>0),
$$

where $a$ is a real number.

In order to prove that a real number $a$ may be found to satisfy eq. (12), we introduce a new function as

$$
f(a)=\sum_{i=1}^{W}\left[1-(q-1)\left(x_{i}-a\right)\right]^{1 /(q-1)} \text {. }
$$

In order to guarantee $f(a) \in R$, 


$$
1-(q-1)\left(x_{i}-a\right) \geqslant 0
$$

must be satisfied. Below, we will discuss the two cases of $0<q<1$ and $1<q$, respectively.

(i) The case of $0<q<1$. In this case eq. (14) can be written as

$$
a \leqslant x_{i}-\frac{1}{(q-1)} .
$$

Since $x_{i}$ is one of the random values among $W$ microstates of the system, one can get the upper limit of the parameter $a$ as

$$
a_{\text {max }} \leqslant x_{\min }-\frac{1}{(q-1)},
$$

where $x_{\min }=\min \left\{x_{i}\right\}$. On the other hand, the derivative of eq. (13) with respect to $a$ gives

$$
f^{\prime}(a)=\sum_{i=1}^{W}\left[1-(q-1)\left(x_{i}-a\right)\right]^{\frac{1}{q-1}-1} .
$$

It is easily seen from eqs. (14) and (17) that $f^{\prime}(a)$ is always positive. It means that $f(a)$ is a monotonically increasing function of $a$ in the region $\left(-\infty, x_{\min }-1 /(q-1)\right)$, as shown in Figure 1, where

$$
\lim _{a \rightarrow-\infty} f(a)=0
$$

and

$$
\begin{aligned}
& \lim _{a \rightarrow x_{\min }-1 /(q-1)}\left[1-(q-1)\left(x_{\min }-a\right)\right]^{\frac{1}{q-1}} \rightarrow+\infty \\
& \Rightarrow \lim _{a \rightarrow x_{\min }-1 /(q-1)} f(a) \rightarrow \infty .
\end{aligned}
$$

It is clearly seen from Figure 1 that the curve of $f(a)$ has a crossing point with the straight line $y=1$. Thus, one can draw a conclusion that eq. (12) has a real number solution $a_{0}$ marked in Figure 1.

(ii) The case of $1<q$. When $1<q$, eq. (14) can be written as

$$
a \geqslant x_{i}-\frac{1}{(q-1)} .
$$

In such a case, the lower limit of $a$ is determined by

$$
a_{\min } \geqslant x_{\max }-\frac{1}{(q-1)},
$$

where $x_{\max }=\max \left\{x_{i}\right\}$. It can be seen from eqs. (14) and (17)

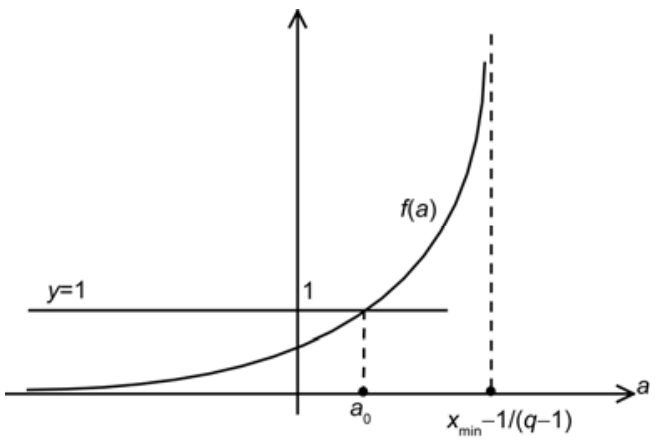

Figure 1 Schematic diagram of $f(a)$ versus $a$ for the case of $0<q<1$. that when $1<q, f(a)$ is also a monotonically increasing function of $a$ in the region $\left(x_{\max }-1 /(q-1),+\infty\right)$, as shown in Figure 2, where

$$
\lim _{a \rightarrow+\infty} f(a) \rightarrow+\infty
$$

and

$$
\begin{aligned}
& \lim _{a \rightarrow x_{\max }-1 /(q-1)} f(a)=\sum_{i=1}^{W}\left[(q-1)\left(x_{\max }-x_{i}\right)\right]^{\frac{1}{q-1}} \\
& \leqslant \sum_{i=1}^{W}\left[(q-1)\left(x_{\max }-x_{\min }\right)\right]^{\frac{1}{q-1}}=W\left[(q-1)\left(x_{\max }-x_{\min }\right)\right]^{\frac{1}{q-1}} .
\end{aligned}
$$

It is seen from Figure 2 that the curve of $f(a)$ has a crossing point with the straight line $y=1$ under the condition

$$
W\left[(q-1)\left(x_{\max }-x_{\min }\right)\right]^{\frac{1}{q-1}} \leqslant 1 .
$$

This indicates that when eq. (24) is satisfied, we can have a real number solution $a_{0}$ for eq. (12).

Substituting eq. (12) into eq. (10) yields

$$
p_{i}=\left[1-(q-1)\left(x_{i}-a\right)\right]^{1 /(q-1)},
$$

and then

$$
x_{i}=\frac{1-p_{i}^{q-1}}{q-1}+a .
$$

From eqs. (8) and (26) one can obtain the uncertainty measure for the $q$-exponential distribution function as

$$
I_{q}=\int \mathrm{d} I=\frac{C_{2}-\sum_{i=1}^{W} p_{i}^{q}}{q(q-1)},
$$

where $C_{2}$ is the integral constant. It should be pointed out that for a determined system, if the condition $p_{i}=\delta_{1, i}$ is chosen, the uncertainty measure should be equal to zero, so one can get $C_{2}=1$. Finally, we have

$$
I_{q}=\frac{1-\sum_{i=1}^{W} p_{i}^{q}}{q(q-1)} \quad(q>0) .
$$

Eq. (28) can be transformed to $I_{\mathrm{BG}}=-\sum_{i=1}^{W} p_{i} \ln p_{i}$ at the $q \rightarrow 1$ limit. This is nothing but the Boltzmann-Gibbs entropy. It can be seen from above analyses that eq. (28) is

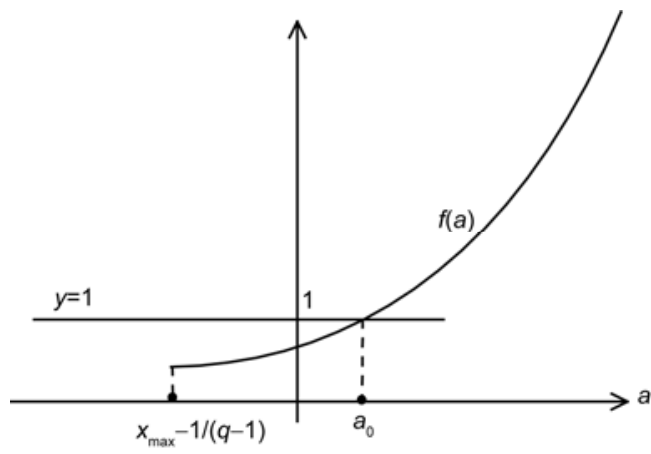

Figure 2 Schematic diagram of $f(a)$ versus $a$ for the case of $1<q$. 
the strict result of eqs. (8) and (10). Therefore, it can be considered as the entropic form of the systems having $q$-exponential distribution.

\section{Discussion}

Eq. (28) is very similar to the Tsallis entropy, except the additional ' $q$ ' in the denominator. However, the properties of this entropy are different from those of the Tsallis one. It can be seen that $\tilde{q} \in R$ in eq. (1), while $I$ in eq. (28) is negative if $q$ is smaller than zero, which does not make sense. Furthermore, eq. (28) can yield exactly the $q$-exponential distribution by the Lagrange multiplier method since the processes of MaxEnt and VarEntropy are self-consistent in mathematics.

The constraints of the probability normalization and energy expectation are $\sum_{i} p_{i}=1$ and $\sum_{i} p_{i} \varepsilon_{i}=U$, where $\left\{\varepsilon_{i}\right\}$ is the energy spectrum of the system. Thus, the Lagrangian can be written as

$$
L=I_{q}-\alpha \sum_{i} p_{i}-\beta \sum_{i} p_{i} \varepsilon_{i},
$$

where $\alpha$ and $\beta$ are two Lagrange multipliers. $\left.\delta L\right|_{p_{i}}=0$ leads to

$$
\begin{aligned}
p_{i} & =\left[(1-q) \alpha-(q-1) \beta \varepsilon_{i}\right]^{\frac{1}{q-1}} \\
& =\left[1-(q-1)\left(x_{i}-a\right)\right]^{\frac{1}{q-1}},
\end{aligned}
$$

where $x_{i}=\beta \varepsilon_{i}$ and $a=1 /(1-q)-\alpha$. Eq. (30) can be transformed to the observable $q$-exponential function by a spectrum shift for the all values of $1>q>0$ and partial values of $q>1$. It is different from eqs. (5)-(7) obtained from the same method but different entropies.

Considering a system with an entropic form described by eq. (28) and only two microstates, the probability of state 1 is $p_{1}$ and the other is $\left(1-p_{1}\right)$. As shown in Figure 3, all the curves are concave.

It means that this entropic form can be maximized for different physical systems with $q>0$. In Figure 3, we have

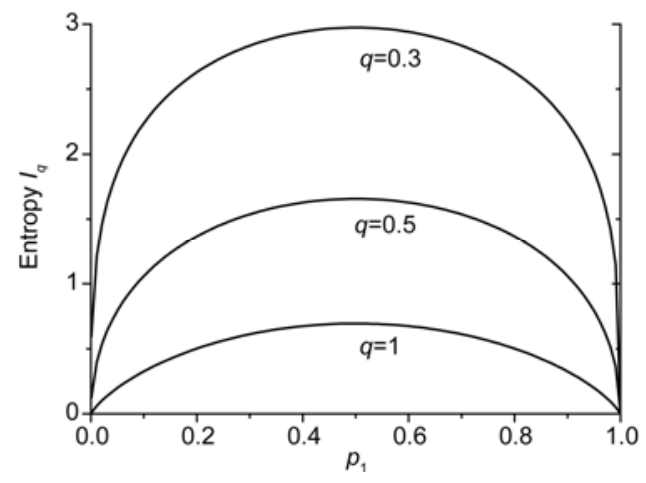

Figure 3 Curves of the entropy of a two state system varing with the probability for the cases of $0<q \leqslant 1$. not presented the cases of $q>1$ since there sometimes does not exist a real number solution for $a$ in eq. (12). This constraint condition is illustrated by eq. (24). Nevertheless, for a system with given spectrum and total number of microstates, if there exist some values of $q(q>1)$ that can satisfy eq. (24) one can also obtain the entropy curves with respect of the probability. The entropies of different $q$ values also keep the concavity, as shown in Figure 4. In comparison, the Tsallis entropy [9], normalized Tsallis entropy [11] and Renyi entropy [13] are not always concave for all possible $q$ values.

For a composed system $C$ consisting of two independent subsystems $A$ and $B$, which have the probability distribution $\left\{p_{i}(A) \mid i=1,2, \ldots W_{A}\right\}$ and $\left\{p_{j}(B) \mid j=1,2, \ldots W_{B}\right\}$, respectively, the independence between the subsystems means that $p_{i, j}(A \mid B)=p_{i}(A) p_{j}(B)$ and then the entropy of the total system $C$ can be written as

$$
I_{q}(C)=I_{q}(A)+I_{q}(B)-q(q-1) I_{q}(A) I_{q}(B) .
$$

The third item on the right hand side of eq. (31) means the nonadditivity of this entropic form, which is similar to that of the Tsallis entropy and will disappear when $q \rightarrow 1$.

\section{Conclusions}

Using the VarEntropy method, we obtain the entropy for the $q$-exponential distribution function which has been observed in more and more complex physical systems presenting long range interactions and/or long-duration memory. This entropy can yield exactly the $q$-exponential distribution function by the Lagrange multiplier method. The traditional Boltzmann-Gibbs entropy can be covered at the $q \rightarrow 1$ limit and the nonadditive item in eq. (31) disappears in this case. It is worth to point out that this entropy is always concave for different values of the nonextensive parameter $q>0$. It means that it is reasonable to adopt this entropy to evaluate the uncertainty (or information) of the systems having observable $q$-exponential distributions.

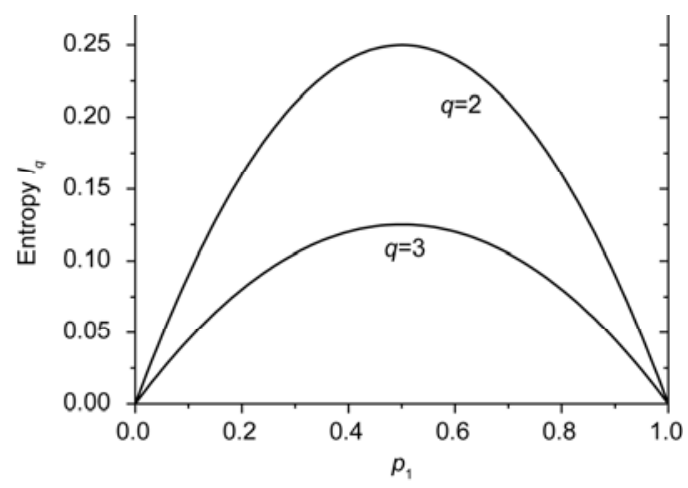

Figure 4 Curves of the entropy of a two state system varing with the probability for the cases of $q>1$. 
This work was supported by the National Natural Science Foundation of China (11005041), Natural Science Foundation of Fujian Province (2010J05007), Scientific Research Foundation for the Returned Overseas Chinese Scholars, Fundamental Research Funds for the Central Universities (JB-SJ1005) and Programme of Introducing Talents of Discipline to Universities (B08033).

1 Huang X P, Driscoll C F. Relaxation of 2D turbulence to a metaequilibrium near the minimal enstrophy state. Phys Rev Lett, 1994, 72: 2187-2191

2 Huang X P, Anderegg F, Hollmann E M, et al. Steady-state confinement of non-neutral plasmas by rotating electric fields. Phys Rev Lett, 1997, 78: 875-878

3 Huang X P, Driscoll C F, Hollmann E M, et al. Test particle transport due to long range interactions. Phys Rev Lett, 1997, 78: 2128-2131

4 Liu B, Goree J. Superdiffusion and non-Gaussian statistics in a driven-dissipative 2D dusty plasma. Phys Rev Lett, 2008, 100: 055003

5 Liu B, Goree J, Feng Y. Non-Gaussian statistics and superdiffusion in a driven-dissipative dusty plasma. Phys Rev E, 2008, 78: 046403

6 Douglas P, Bergamini S, Renzoni F. Tunable Tsallis distributions in dissipative optical lattices. Phys Rev Lett, 2006, 96: 110601

7 Zheng L, Li W. Thermoequilibrium statistics for a finite system with energy nonextensivity. Chin Sci Bull, 2011, 56: 3666-3670

8 Liu Z P, Guo L N, Du J L. Nonextensivity and the q-distribution of a relativistic gas under an external electromagnetic field. Chin Sci Bull, 2011, 56: 3689-3692

9 Tsallis C. Possible generalization of Boltzmann-Gibbs statistics. J Stat Phys, 1988, 52: 479-487
10 Tsallis C, Mendes R S, Plastino A R. The role of constraints within generalized nonextensive statistics. Physica A, 1998, 261: 534-554

11 Landsberg P T, Vedral V. Distributions and channel capacities in generalized statistical mechanics. Phys Lett A, 1998, 247: 211-217

12 Rajagopal A K, Abe S. Implications of form invariance to the structure of nonextensive entropies. Phys Rev Lett, 1999, 83: 17111714

13 Rényi A. Probability Theory. North-Holland: Amsterdam, 1970

14 Abe S. Anomalous behavior of q-averages in nonextensive statistical mechanics. J Stat Mech, 2009, P07027

15 Abe S. Generalized molecular chaos hypothesis and the H-theorem: Problem of constraints and amendment of nonextensive statistical mechanics. Phys Rev E, 2009, 79: 041116

16 Wang Q A. Incomplete statistics: nonextensive generalizations of statistical mechanics. Chaos Soliton Fract, 2001, 12: 1431-1437

17 Huang Z F, Lin B H, Chen J C. A new expression of the probability distribution in incomplete statistics and fundamental thermodynamic relations. Chaos Soliton Fract, 2009, 40: 1277-1281

18 Wang Q A. Probability distribution and entropy as a measure ofuncertainty. J Phys A: Math Theor, 2008, 41: 065004

19 Ou C J, El Kaabouchi A, Nivanen L, et al. Maximizable informational entropy as measure of probabilistic uncertainty. Int J Mod Phys B, 2010, 24: 3461-3468

20 Ou C J, El Kaabouchi A, Chen J C, et al. Generalized measure of uncertainty and the maximizable entropy. Mod Phys Lett B, 2010, 24: $825-831$

21 Vakarin E V, Badiali J P. A link between the maximum entropy approach and the variational entropy form. Mod Phys Lett B, 2011, 25: $1821-1828$

Open Access This article is distributed under the terms of the Creative Commons Attribution License which permits any use, distribution, and reproduction in any medium, provided the original author(s) and source are credited. 